\title{
Specificity and sensitivity assessment of selected nasal provocation testing techniques
}

\author{
Edyta Krzych-Fałta ${ }^{1}$, Konrad Furmańczyk ${ }^{1,2}$, Bolesław Samoliński ${ }^{1}$
}

${ }^{1}$ Unit of Environmental Hazard Prevention and Allergology, Faculty of Health Science, Medical University of Warsaw, Warsaw, Poland ${ }^{2}$ Chair of Applied Mathematics, Faculty of Applied Informatics and Mathematics, Warsaw University of Life Sciences, Warsaw, Poland

Adv Dermatol Allergol 2016; XXXIII (6): 464-468

DOI: 10.5114/pdia.2016.61339

\begin{abstract}
Introduction: Nasal provocation testing involves an allergen-specific local reaction of the nasal mucosa to the administered allergen.

Aim: To determine the most objective nasal occlusion assessment technique that could be used in nasal provocation testing.

Material and methods: A total of 60 subjects, including 30 patients diagnosed with allergy to common environmental allergens and 30 healthy subjects were enrolled into the study. The method used in the study was a nasal provocation test with an allergen, with a standard dose of a control solution and an allergen (5,000 SBU/ml) administered using a calibrated atomizer into both nostrils at room temperature. Early-phase nasal mucosa response in the early phase of the allergic reaction was assessed via acoustic rhinometry, optical rhinometry, nitric oxide in nasal air, and tryptase levels in the nasal lavage fluid.

Results: In estimating the homogeneity of the average values, the Levene's test was used and receiver operating characteristic curves were plotted for all the methods used for assessing the nasal provocation test with an allergen. Statistically significant results were defined for $p<0.05$. Of all the objective assessment techniques, the most sensitive and characteristic ones were the optical rhinometry techniques (specificity $=1$, sensitivity $=1$, $\mathrm{AUC}=1, \mathrm{PPV}=1$, NPV = 1).

Conclusions: The techniques used showed significant differences between the group of patients with allergic rhinitis and the control group. Of all the objective assessment techniques, those most sensitive and characteristic were the optical rhinometry.
\end{abstract}

Key words: nasal provocation test, receiver operating characteristic.

\section{Introduction}

Due to its high specificity and sensitivity, nasal provocation testing (NPT) is widely used in differential diagnostics of allergic rhinitis (AR). Nasal provocation testing allows for "recreating the response of the upper airways to natural exposure to allergens or irritants and doing research into the pathophysiology of the upper airways by testing potential biochemical mediators" (Committee for Upper Airways Allergies) [1, 2]. Although the early and late phases of an allergic reaction have a fairly typical course, the NPT techniques used in diagnostics continue to be disputed due to insufficient data for establishing reference values in attempts to standardize the methods.
These as yet not standardized NPT techniques include acoustic rhinometry (ARM), optical rhinometry (ORM), rhinomanometry, peak nasal inspiratory flow (PNIF) assessment, nasal nitric oxide (nNO) concentration measurement, and inflammatory marker assessment in the nasal lavage fluid.

\section{Aim}

The aim of this study was to determine the most objective technique for examining nasal occlusion that could be used in nasal provocation testing.

Address for correspondence: Edyta Krzych-Fałta PhD, Unit of Environmental Hazard Prevention and Allergology, Faculty of Health Science, Medical University of Warsaw, 1A Banacha St, 02-097 Warsaw, Poland, phone: +48 2259910 40, fax: +48 225992042 , e-mail: e.krzych@gmail.com Received: 2.02.2016, accepted: 1.04.2016. 


\section{Material and methods}

The study population comprised 60 subjects: 30 patients (diagnosed with allergic rhinitis (AR) to common environmental allergens: dust mites, grasses) and 30 healthy controls $(\mathrm{HC})$ with no symptoms of allergy (Figure 1). Subjects were qualified for the study based on documented history of allergy to a given allergen, a positive skin-prick test, computed tomography scans of paranasal sinuses that excluded inflammation, preserved patency of the osseous part of nasal passages. The diagnostic technique used in the study was NPT with $0.9 \%$ saline and $0.4 \%$ phenol - as a control solution and 5,000 standardized biological units SBU/ml (Allergopharma) administered with a calibrated atomizer into both nostrils at room temperature at a dose of $0.2 \mathrm{ml}$. The nasal reaction was assessed via light extinction curve analysis of infrared light emitted and detected by ORM, ARM, $n N O$ concentration measurement, tryptase (TR) concentration measurement as well as via a visual analog scale (VAS) at 5, 10, 15, and 20 min after allergen provocation.

Symptom severity was assessed via a Visual Analog Scale (VAS) (a 10-cm horizontal line representing the intensity of discomfort as a percentage). The evaluated symptoms included nasal congestion, rhinorrhea, and nasal irritation, with a predetermined positive reaction cut-off point of 20\%. Optical rhinometry (Rhios GmbH, Grosserkmannsdorf, Germany) was used for direct, continual assessment of changes (optical density - OD) in the volume of blood flowing through nasal vessels with the use of an emitter and a sensor placed on the opposite sides of the bridge of the nose. The threshold optical density level considered to be a positive response was $\geq 0.2$ OD [3]. Another technique used in assessing the nasal mucosa response to topical allergen application was ARM (Rhinometrics, Denmark). The selected crosssectional area (CSA-2) on an ARM curve was used to as- sess nasal edema severity separately in the right and left nasal cavities, via the relative reactivity formula:

$$
\operatorname{Re}=\frac{(\text { CSAll }- \text { CSAI })}{\text { CSAI }} \times 100 \% \text {, }
$$

where: CSAI - cross-sectional area before allergen provocation, CSAll - cross-sectional area after allergen provocation. If an edematous reaction develops, the value of reactivity becomes negative [4].

The predetermined relative reactivity threshold of the nasal mucosa was set at 20\%. nNO values (ExpAir, Medisoft, Belgium) were measured electrochemically within the range of $0.1-6,000 \mathrm{ppb}$ and presented in the form of three reproducible, normal nNO concentration curves [5]. Nasal lavage fluid was collected in room conditions according to Greiff et al. [6].

The study was conducted using funds received as a supervisor grant from the Ministry of Science and Higher Education (No. N402 520839) and was approved by the Institutional Review Board at the Medical University of Warsaw (KB/79/2009).

\section{Statistical analysis}

Student's t-test and Pearson's correlation coefficient were calculated in order to determine the relationship between variables and the strength of the relationship. In estimating the homogeneity of the average values, the Levene's test was used and receiver operating characteristic (ROC) curves were plotted for all the methods used for assessing the nasal provocation test with an allergen. $P$-value $<0.05$ was considered to be statistically significant.

\section{Results}

After contact with the mucous membrane of the nasal cavity, the allergen caused a number of events affecting the early phase of the allergic reaction. The

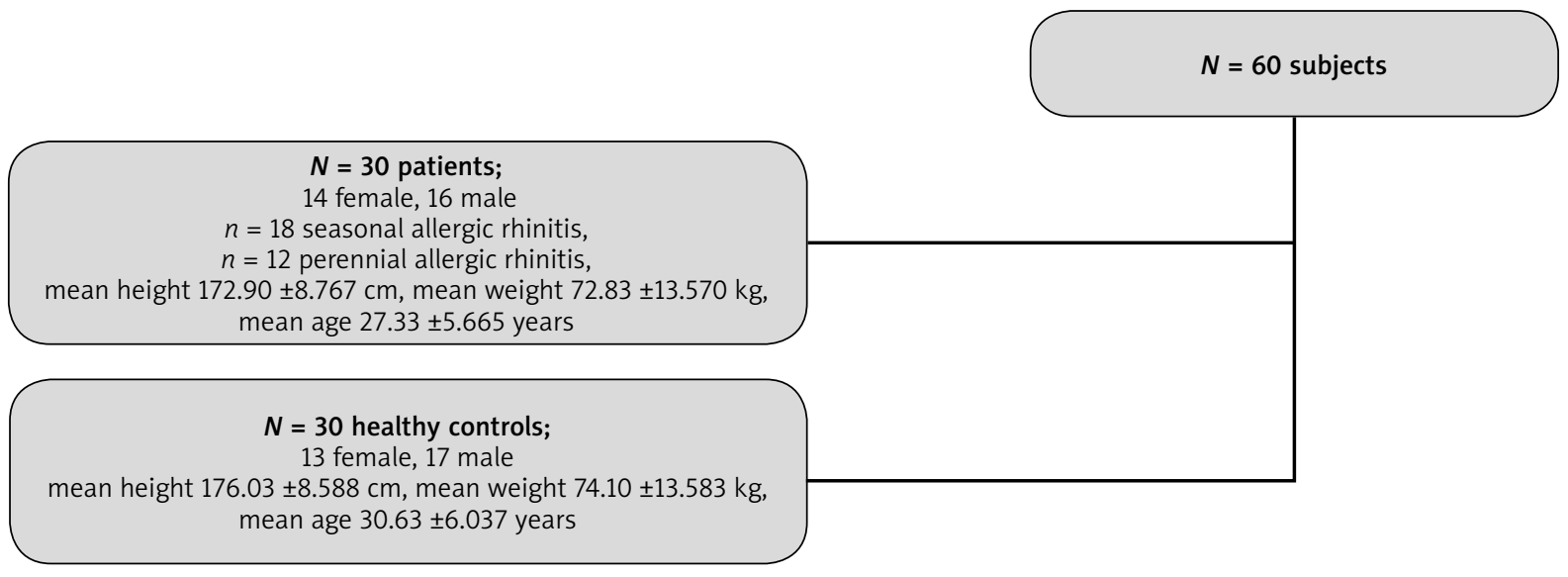

Figure 1. Characteristic group 
beginning of the reaction was recorded at minute 3.15 of the test on an OR curve (light extinction) returned to its lowest (baseline) level after $28.15 \mathrm{~min}$. The mean value of infrared light absorption in the AR group in comparison with that in the control group was $\mathrm{OD}=0.431$, $(p<0.05)$ accompanied by nasal itching (more intense in the case of the patients with chronic rhinitis $(p=0.042)$ compared to the patients with seasonal rhinitis) with an increased number of sneezes [7]. At minute 10 of the test, nasal secretion increased and nasal obstruction was increasingly intense and remained at a high level during the early phase of the allergic reaction. Increased reactivity of the mucous membrane and a change, over time, of the minimal cross-sectional area of the nasal cavity in the region of the head of the inferior nasal concha (CSA-2, reactivity $-21.97 \%$ ) were observed at minute 15 and 20 of the test in the groups of patients with AR [8]. The intensifying nasal obstruction was accompanied by a reduction in the concentration of nitrogen oxide in the air exhaled from the upper airways (from $1253.42 \mathrm{ppb}$ to $927.83 \mathrm{ppb}$ on average in the case of the chronic rhinitis patients and from 1059.44 to $847.33 \mathrm{ppb}$ in the seasonal rhinitis group) [9], with an increase in the concentration of tryptase $(2.16 \mu \mathrm{g} / \mathrm{I}$ within the chronic rhinitis group and $2.39 \mu \mathrm{g} / \mathrm{l}$ within the seasonal rhinitis group) in the nasal lavage fluid. As regards non-nasal symptoms, coughing was observed especially among the chronic rhinitis patients at minute $15(p=0.044)$ and at minute $20(p=$ 0.040) of the test. We observed significant differences between study groups in terms of nasal symptoms such as nasal irritation; rhinorrhea, nasal congestion, number of sneezes in the period from minute 5 to $20(p<0.05)$.

The two-dimensional graph of the ROC curve showed the highest specificity and sensitivity for the following: VAS (specificity $=1$, sensitivity $=1$, area under the curve $(\mathrm{AUC})=1$, positive predictive value $(\mathrm{PPV})=1$, negative predictive value $(N P V)=1)$, ORM (specificity $=1$, sensitivity $=1, \mathrm{AUC}=1, \mathrm{PPV}=1, \mathrm{NPV}=1$ ), nasal lavage TR levels (specificity $=0.97$, sensitivity $=0.96$, AUC $=0.995$, PPV $=0.96, \mathrm{NPV}=0.97)$. The remaining two NPT techniques, i.e. $A R M$ (for the right side: specificity $=0.71$, sensitivity $=0.73, \mathrm{AUC}=0.716$, PPV $=0.71, \mathrm{NPV}=0.72$; for the left side: specificity $=0.6$, sensitivity $=0.77, \mathrm{AUC}=0.69$, PPV $=0.66, \mathrm{NPV}=0.7277$ ) and $\mathrm{nNO}$ (specificity $=0.73$, sensitivity $=0.57, \mathrm{AUC}=0.67$, PPV $=0.68, \mathrm{NPV}=0.63$ ) allowed for only a moderate degree of differentiation between the healthy and the affected individuals (Figure 2).

\section{Discussion}

Nasal provocation testing involves an allergen-specific local reaction of the nasal mucosa to the administered allergen. Irrespective of the assessment technique used, NPT is characterized by particularly high specificity and sensitivity (specificity $=83.7 \%$ and sensitivity $=100 \%$ ) [10]. Unsel et al. conducted NPT with latex in a group of
94 individuals $(n=29$ patients with a positive skin-prick test to latex, $n=35$ a randomized group with allergies to non-latex allergens, and $n=30$ healthy individuals) and demonstrated a positive reaction in the allergic groups at latex concentrations of merely $0.05 \mu \mathrm{g} / \mathrm{ml}$. The specificity (96\%), sensitivity (100\%), NPV 98\%, and PPV 100\% of an NPT with a standardized allergen were considerably higher than those with a latex glove test, at sensitivity of $81 \%$, specificity of $90 \%$ with NPV of $75 \%$ and PPV of 93\%, respectively [11].

Depending on the assessment technique or test type (specific, non-specific) NPT yields various ROC curve values. The most extensively researched NPT technique is ARM. The level of nasal mucosal response to local application of an allergen is assessed based on the minimum cross-sectional area (MCA) typically represented by the I-notch (isthmus nasi) before nasal allergen application or the C-notch (concha) after nasal allergen application, relevant cross-sectional areas (CSAs) of the nasal cavity from an acoustic rhinometry curve, relative reactivity, and total nasal volume or the left and right side of the nasal cavity separately.

Jang et al. conducted a study in a group of 262 patients (group $1, n=110$, included subjects with negative skinprick tests to house dust mites; group $2, n=53$, subjects with moderately positive tests; group $3, n=99$, subjects allergic to house dust mites) and found group 3 to differ significantly from the other two groups in terms of NPT results $(p<0.01)$. An analysis of the ROC curve for VAS, with the reaction threshold of $6.5 \mathrm{pts}$, demonstrated specificity of $90.5 \%$ and sensitivity of $77.4 \%$. Acoustic rhinometry conducted with nasal patency reduced to $27.6 \%$ had an estimated sensitivity of $73.4 \%$ and specificity of $58.1 \%$ [12].

Kim et al. used ARM to assess non-specific nasal provocation with cold air in a group of 45 patients diagnosed with cold air hypersensitivity and in 53 individuals without evidence of cold air hypersensitivity and demonstrated significantly more pronounced nasal changes (total nasal symptom score - TNSS) in the hypersensitive group $(p<0.01)$. The specificity and sensitivity of the ROC curve with a 1.5-point TNSS threshold was $86.8 \%$ and $75.6 \%$, respectively. We achieved a relatively high sensitivity and specificity for predicted mucosal reactivity levels of $15 \%$ (sensitivity $93.3 \%$ and specificity $77.4 \%$ ) and $19.5 \%$ (sensitivity $84.4 \%$ and specificity $77.4 \%$ ) [13].

This analysis also demonstrated a significantly high level of specificity and sensitivity of ARM in comparison to nNO concentration assessment, but significantly lower than that of ORM (100\% specificity and $100 \%$ sensitivity) and VAS. Optical rhinometry is a relatively new technique, used not only in nasal allergy diagnostics [14] but also as an objective assessment of the effectiveness of treatment and physiological changes in the nasal cavity. There is only one publication documenting the specificity and sensitivity of ORM versus ARM in the literature on the subject. A study by Agarwal et al. conducted in a group of 

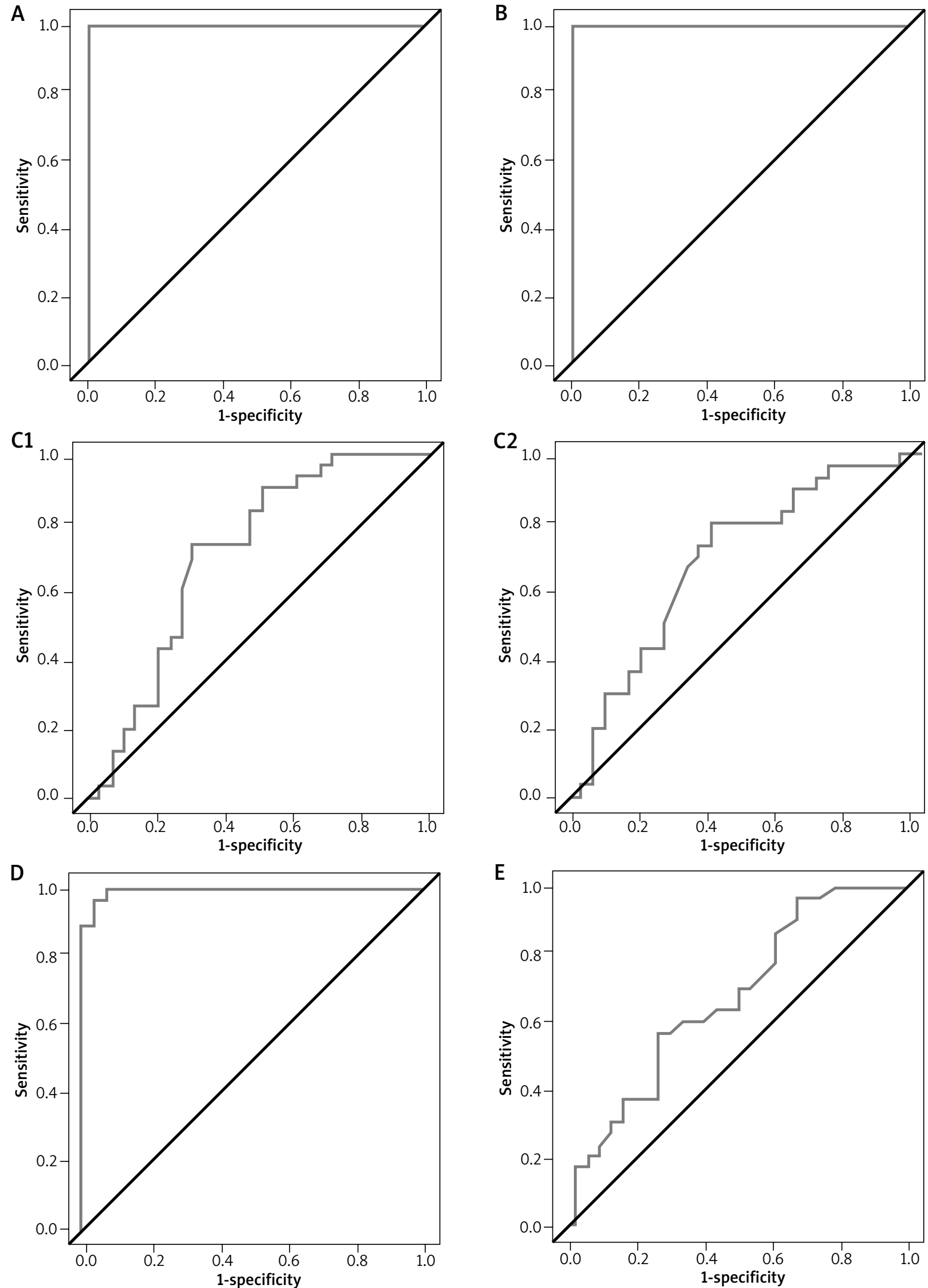

Figure 2. Specificity and sensitivity of selected techniques for assessing the early phase of an allergic reaction: $\mathrm{A}-$ visual analogue scale, B - optical rhinometry, C - acoustic rhinometry (C1 - right side, C2 - left side), D - tryptase in the nasal lavage fluid, E - nasal nitric oxide 
22 subjects (11 patients diagnosed with AR and 11 healthy individuals, mean age: $29 \pm 9$ years) assessed the specificity and sensitivity of NPT with two allergens: Alternaria ( $n=8$ subjects) and Aspergillus $(n=7)$. Nasal provocation testing with Alternaria showed a higher VAS specificity ( $A \cup C=0.76, p<0.05$ for VAS) in comparison with Aspergillus (AUC $=0.70, p=0.14$ for VAS). Conversely, the study groups did not differ significantly in terms of ORM and ARM results (Alternaria; $A U C=0.48, p=0.9$ for ORM and $\mathrm{AUC}=0.74, p=0.8$ for ARM and Aspergillus ORM 0.53, $p=0.81$ and ARM 0.42, $p=0.05)$ [15].

Other NPT techniques, not qualified as objective techniques in the assessment of the ROC curve showed a moderate specificity and sensitivity in differentiating those affected from healthy individuals. Due to high bioactivity of $\mathrm{NO}$, nNO is mainly used in screening for primary ciliary dyskinesia. An allergen challenge chamber study by Hohifeld et al. assessed the specificity and sensitivity of nNO in a group of 60 patients diagnosed with $A R$ and 60 healthy individuals. The area under the ROC curve demonstrated a significant superiority of assessing nasal symptoms via TNSS (0.86) in comparison with nNO $(A \cup C=0.75)[16]$. A study by Tworek et al. in a group of 24 allergic patients demonstrated $\mathrm{nNO}$ specificity of 1.0 and sensitivity of 0.609 (PPV $=1.0, \mathrm{NPV}=0.71, \mathrm{AUC}=0.814)$ with the reported change in nNO by $11.987 \%$ [17].

The practicality of evaluating inflammatory markers, such as TR levels, in the nasal lavage fluid is limited due to the limited availability and the high expenses associated with enzyme-linked immunosorbent assays. Our own observations revealed only moderate sensitivity and specificity of this technique in NPT. We observed an approximately three-fold or greater increase in tryptase levels in the nasal lavage fluid, the latter significantly different from the baseline measurement. Measuring TR levels is particularly useful in diagnosing an anaphylactic shock; with an 8.23-ng/ml increase showing high sensitivity of $94.12 \%$ and specificity of $92.31 \%$ in in vitro studies via immunofluorescence $[18,19]$.

\section{Conclusions}

The techniques used showed significant differences between the group of patients with allergic rhinitis and the control group. Of all the objective assessment techniques, those most sensitive and characteristic were the optical rhinometry.

\section{Conflict of interest}

The authors declare no conflict of interest.

\section{References}

1. Malm L, Gerth van Wijk R, Bachert C. Guidelines for nasal provocations with aspects on nasal patency, airflow and airflow resistance. Rhinology 2000; 38: 1-6.
2. Naclerio RM, Meier HL, Kagey-Sobotka A, et al. Mediator release after nasal airway challenge with allergen. Am Rev Respir Dis 1983; 128: 597-602.

3. Hampel U, Schleicher E, Wüstenberg EG, et al. Optical measurement of nasal swelling. IEEE Trans Biomed Eng 2004; 51: 1673-9.

4. Samoliński B. Analiza wyników rynometrii akustycznej na potrzeby diagnostyki rynoalergologicznej. Scholar, Warsaw 1998.

5. ATS/ERS recommendations for standardized procedures for the online and offline measurement of exhaled lower respiratory nitric oxide and nasal nitric oxide. Am J Respir Crit Care Med 2005; 171: 912-30.

6. Greiff L, Pipkorn U, Alkner U, et al. The nasal pool device applies controlled concentrations of solutes on human nasal airway mucosa and samples its surface exudations/secretions. Clin Exp Allergy 1999; 20: 253-9.

7. Krzych-Fałta E, Sybilski A, Wojas O, et al. Optical rhinometry in nasal provocation test. Postep Derm Alergol 2015; 32: 449-54.

8. Krzych-Fałta E, Furmańczyk K, Samoliński B. Najmniejsze przekroje poprzeczne jamy nosa w ocenie donosowej próby prowokacyjnej z alergenem. Alergol Pol 2016; 3: 108-13.

9. Kłak A, Krzych-Fałta E, Samolinski B, et al. Diagnostic significance of nitric oxide exhaled air from the airways in allergic rhinitis patients. Adv Dermatol Allergol 2016; 33: 87-95.

10. de Blay F, Doyen V, Lutz C, et al. A new, faster, and safe nasal provocation test method for diagnosing mite allergic rhinitis. Ann Allergy Asthma Immunol 2015; 115: 385-90.

11. Unsel M, Mete N, Ardeniz O, et al. The importance of nasal provocation test in the diagnosis of natural rubber latex allergy. Allergy 2009; 64: 862-7.

12. Jang TY, Kim YH. Nasal provocation test is useful for discriminating allergic nonallergic, and local allergic rhinitis. Am J Rhinol Allergy 2015; 29: 100-4.

13. Kim YH, Jang TY. Diagnostic criteria of nonspecific hyperreactivity using cold dry air provocation with acoustic rhinometry. Otolaryngol Head Neck Surgery 2011; 144: 91-5.

14. Luong A, Cheung EJ, Citardi MJ, et al. Evaluation of optical rhinometry for nasal provocation testing in allergic and nonallergic subject. Otolaryngol Head Neck Surgery 2010; 143: 284-9.

15. Agarwal G, Hernandez D, Citardi MJ, et al. End-organ testing for allergic rhinitis with fungi is poorly correlated with fungal sensitivity. Otolaryngol Head Neck Surgery 2013; 148: 391-5.

16. Hohifeld JM, Holland-Letz T, Larbig M, et al. Diagnostic value of outcome measures following allergen exposure in an environmental challenge chamber compared with natural conditions. Clin Exp Allergy 2010; 40: 998-1006.

17. Tworek D, Kuna P. Nasal nitric oxide measurements in the assessment of nasal allergen challenge. J Investig Allergol Clin Immunol 2012; 22: 102-8.

18. De Schryver S, Halbrich M, Clarke A. Tryptase levels in children presenting with anaphylaxis: temporal trends and associated factors. J Allergy Clin Immunol 2016; 137: 1138-42.

19. Enrique E, García-Ortega P, Sotorra O, et al. Usefulness of UniCAP-Tryptase fluoroimmunoassay in the diagnosis of anaphylaxis. Allergy 1999; 54: 602-6. 